\title{
Cell death-based treatment of lung adenocarcinoma
}

\author{
Tatiana V. Denisenko ${ }^{1}$, Inna N. Budkevich ${ }^{1}$ and Boris Zhivotovsky ${ }^{1,2}$
}

\begin{abstract}
The most common type of lung cancer is adenocarcinoma (ADC), comprising around $40 \%$ of all lung cancer cases. In spite of achievements in understanding the pathogenesis of this disease and the development of new approaches in its treatment, unfortunately, lung ADC is still one of the most aggressive and rapidly fatal tumor types with overall survival less than 5 years. Lung ADC is often diagnosed at advanced stages involving disseminated metastatic tumors. This is particularly important for the successful development of new approaches in cancer therapy. The high resistance of lung ADC to conventional radiotherapies and chemotherapies represents a major challenge for treatment effectiveness. Here we discuss recent advances in understanding the molecular pathways driving tumor progression and related targeted therapies in lung ADCs. In addition, the cell death mechanisms induced by different treatment strategies and their contribution to therapy resistance are analyzed. The focus is on approaches to overcoming drug resistance in order to improve future treatment decisions.
\end{abstract}

\section{Facts}

- Lung adenocarcinoma is one of the most aggressive and rapidly fatal tumor types.

- Resistance of lung adenocarcinomas to conventional radio- and chemotherapies represents a major challenge for treatment effectiveness.

- Combined therapies overcome resistance and are more effective than drugs targeting only one specific protein or pathway.

\section{Open questions}

- What is the role of driving mutations in targeting therapy for lung adenocarcinoma?

- What should be done to improve the outcome of patients with tumors harboring specific alterations?

- Is crosstalk between different cell death modalities

\footnotetext{
Correspondence: Boris Zhivotovsky (Boris.Zhivotovsky@ki.se)

${ }^{1}$ Faculty of Medicine, MV Lomonosov Moscow State University, 119991

Moscow, Russia

${ }^{2}$ Institute of Environmental Medicine, Division of Toxicology, Karolinska

Institutet, Box 210, Stockholm SE-171 77, Sweden

Edited by M. Piacentini
}

significant in combating lung adenocarcinoma?

- How can the resistance of lung adenocarcinoma to therapy be overcome?

\section{Introduction}

Cancer comprises a highly heterogeneous and complex set of diseases associated with a variety of genetic and epigenetic aberrations. The "hallmarks of cancer" involve a set of cellular traits essential for malignant transformation and tumor maintenance. Among these are sustained proliferative signaling, induced angiogenesis, activation of invasion and metastasis, resistance to cell death, ability to escape immunological surveillance, and various others ${ }^{1,2}$. Genetic intra-tumor heterogeneity also can contribute to treatment failure and drug resistance. Despite extensive research, the intrinsic and acquired resistance of tumors to drug treatment remains a fundamental challenge in improving patient' outcomes.

Lung cancer (LC) is the leading cause of cancer-related mortality ${ }^{3}$. Based on histology, LC is divided into two main subtypes: small cell lung carcinoma (SCLC) and non-small-cell lung carcinoma (NSCLC), accounting for 15 and $85 \%$ of all cases, respectively ${ }^{4}$. NSCLC is further 
classified into three types: squamous-cell carcinoma, adenocarcinoma, and large-cell carcinoma. Squamous-cell carcinoma comprises $25-30 \%$ of all LC cases. It arises from early versions of squamous cells in the airway epithelial cells in the bronchial tubes in the center of the lungs. The most common type of $\mathrm{LC}$ is adenocarcinoma (ADC), which comprises around $40 \%$ of all LC. Lung ADCs develop from small airway epithelial, type II alveolar cells, which secrete mucus and other substances ${ }^{5,6}$. Large-cell (undifferentiated) carcinoma accounts for $5-10 \%$ of LC. This type of carcinoma shows no evidence of squamous or glandular maturation and as a result is often diagnosed by default through the exclusion of other possibilities ${ }^{7}$. The discovery of mutated oncogenes, which encode activated signaling molecules that drive cellular proliferation and promote tumor growth, has now led to the development of more effective and less toxic targeted drugs for LC patients. However, similar to conventional chemotherapies, these new-targeted drugs also have a propensity to fail due to the development of resistance. Gene mutations and focal amplification are genetic changes that modulate the sensitivity of tumors to the induction of cell death, and, therefore, differences in treatment sensitivity may depend on the susceptibility of LC cells, in general, and lung ADC cells, in particular, to undergo cell death ${ }^{8}$.

Here we discuss recent advances in understanding the molecular pathways driving tumor progression and related targeted therapies in lung ADCs. In addition, the cell death mechanisms induced by different treatment strategies and their contribution to therapy resistance are analyzed. The focus is on the approaches to overcoming drug resistance in order to improve future treatment decisions.

\section{Driving mutations}

Lung ADCs commonly contain a heterogeneous mixture of histological growth patterns, classified as "mixed type" ${ }^{\prime \prime}$. Although histological features and marker expression remain the basis of clinical diagnosis, recent advances in sequencing technologies have led to an understanding of tumor heterogeneity and have allowed the further subdivision of lung ADC into molecular subsets according to a classification based on so-called driver mutations. These mutations represent molecular alterations essential for tumor initiation and growth. They can often be detected in genes that control cellular proliferation and survival ${ }^{10,11}$. Thus, tumors might rely on the expression of these single-mutant oncogenes to promote tumor growth and survival, also known as the concept of oncogene addiction ${ }^{12,13}$. As tumor cells depend on the aberrant activity of a specific mutated gene or pathway for survival and proliferation, their inactivation is generally sufficient to induce growth arrest and/or cell death ${ }^{14}$. An interesting hypothesis has been proposed to explain the phenomenon of oncogenic addiction. According to this hypothesis, the apoptotic response observed in tumors in the case of acute disruption of an oncogene product results from differential decay of several pro-survival and pro-apoptotic signals emanating from the oncoproteins ${ }^{15}$. The disturbance in the balance between pro-apoptotic and pro-survival signals could trigger oncogenic shock, which eventually might drive tumor cell death ${ }^{15,16}$.

The first actionable mutation detected in lung ADC was mutation in epidermal growth factor receptor (EGFR), a transmembrane receptor tyrosine kinase (RTK) that represents either somatic mutation deletion in exon 19 or L858R point mutation (Fig. 1) ${ }^{15,17}$. EGFR mutations near the ATP cleft of the tyrosine kinase (TK) domain result in increased receptor activation and act as oncogenic drivers. Binding with ligands (EGF and TGF- $\alpha$ ) leads to conformational changes in EGFR and homodimerization or hetero-dimerization with other human epidermal growth factor receptor (HER) family members. There is subsequent auto-phosphorylation of the cytoplasmic TK domain with the help of adapter proteins (e.g., SHC and GRB-2), triggering downstream signaling pathways: (1) the rat sarcoma (RAS)/rapidly accelerated fibrosarcoma (RAF)/mitogen-activated protein kinase (MAPK) pathway; (2) the phosphatidylinositol-3-kinase (PI3K)/protein kinase B (AKT) pathway; (3) the Janus kinase (JAK)/signal transducers and activators of transcription (STAT) pathway. This stimulates mitosis, leading to cell proliferation and inhibition of apoptosis ${ }^{18,19}$. These pathways are crucial for normal cell growth. EGFR also serves as a stimulus for cancer growth.

Several types of activating mutations are known to occur in EGFR in NSCLC: Class I exon 19 in-frame deletions (44\% of all EGFR mutations), Class II single amino acid changes (L858R 41\%, G719 4\%, other missense mutations 6\%), and Class III exon 20 in-frame duplication/insertions (5\%). All these mutations occur in the TK domain of EGFR. In $85 \%$ of all EGFR-activating mutations are exon 19 in-frame deletions or L858R, and they tend to be sensitive to currently approved EGFR inhibitors ${ }^{20}$. Class III mutations are generally insensitive to EGFR inhibitors with the exception of A763_Y764insFQEA ${ }^{21,22}$.

The next actionable genetic abnormality detected in NSCLC was the anaplastic lymphoma kinase (ALK) fusion oncogene, which is characterized by a fusion between echinoderm microtubule-associated protein-like 4 (EML4) and anaplastic lymphoma kinas (ALK). This fusion generates an overexpressed and activated TK, whereas normal lung tissue does not express ALK. EML4-ALK fusions are found in around $3-13 \%$ of lung ADC patients, and are largely mutually exclusive with alterations in other RTKs or KRAS based on analysis of almost 1700 tumors $^{23}$.

KRAS is the most commonly mutated oncogene in lung ADC, with mutations detected in around 30\% of 


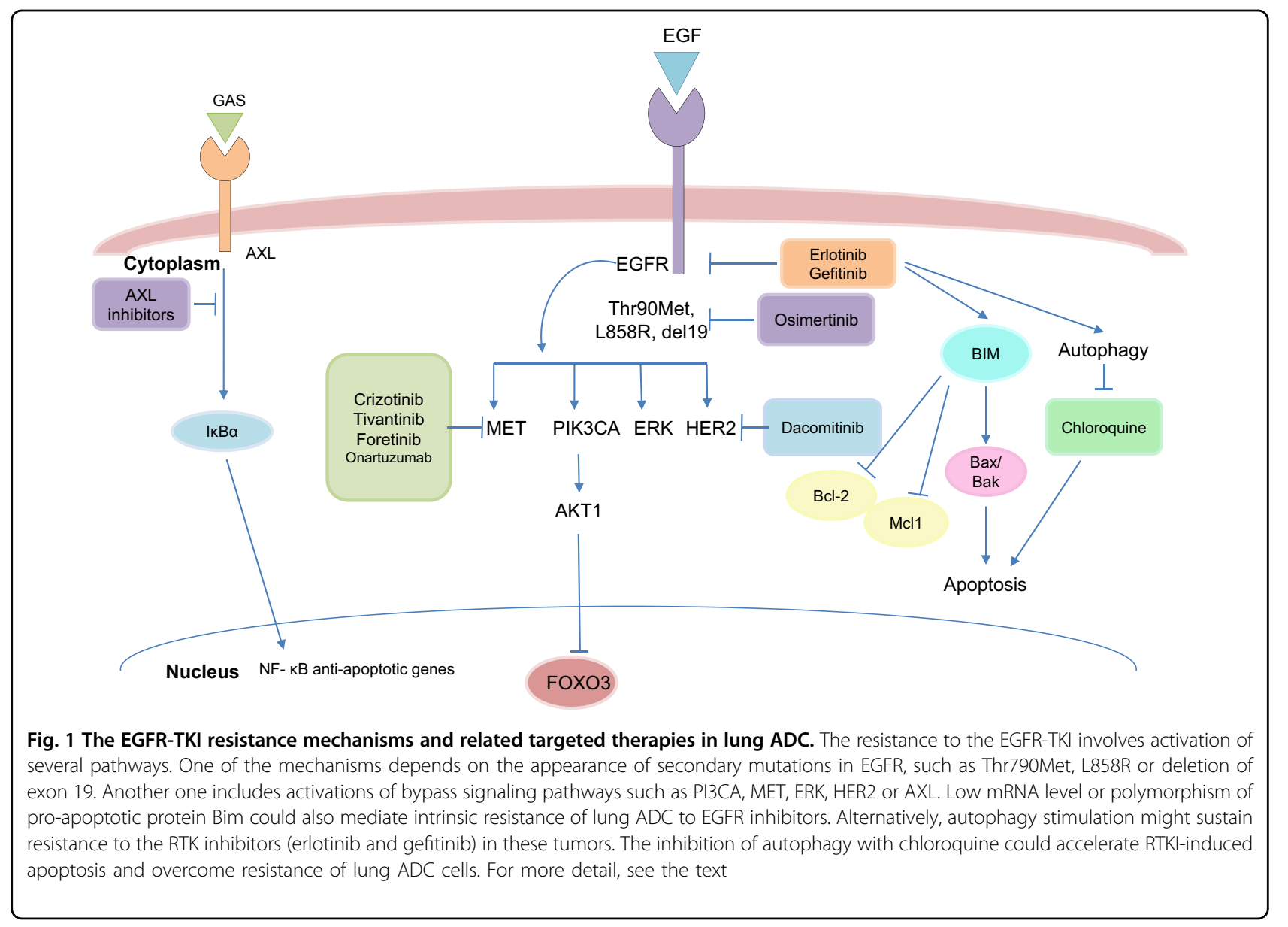

patients $^{23}$. Almost 97\% of KRAS mutations in lung ADCs result in amino-acid substitution at codon 12 and 13 . The mutated KRAS proteins exhibit impaired GTPase activity, resulting in constitutive activation of RAS signaling ${ }^{24}$. The presence of EGFR and KRAS mutations is also mutually exclusive in the same tumor. The role of KRAS mutational status as a marker of response to standard chemotherapy alone in NSCLC is poorly understood, but it has been clearly demonstrated that the occurrence of KRAS mutations is associated with the shortest survival of NSCLC patients treated with platinum-based and antiEGFR therapies ${ }^{25,26}$.

Another targetable mutation in lung ADCs is c-MET, which also belongs to RTKs ${ }^{27}$. Binding with its ligand, hepatocyte growth factor (HGF), triggers receptor dimerization and phosphorylation, leading to conformational changes of c-MET that activate the TK domain as well as a wide range of different cellular signaling pathways, including those involved in proliferation, motility, migration, and invasion. Although c-MET is important for the control of tissue homeostasis under normal physiological conditions, it has also been found to be aberrantly activated in human cancers via gene mutation, amplification or protein overexpression ${ }^{19}$.

In addition, several other NSCLC driver mutations/ gene translocations are currently under investigation, including ROS1/RET rearrangements, and BRAF/ PIK3CA and HER2/MEK mutations, all of which might undergo specific targeted therapy ${ }^{17}$. However, it is remarkable, that in up to $40 \%$ of lung ADCs no driving mutations could be identified despite routinely used molecular diagnostics ${ }^{28,29}$.

\section{Targeted therapies}

The identification of driver mutations in lung ADCs has led to the development of effective personalized treatment strategies (Table 1). Targeting the EGFR pathway represents a pioneering approach to personalized medicine in LC. Recently, a variety of TK inhibitors (TKIs) targeting EGFR have been tested in clinical trials and approved by the FDA ${ }^{30,31}$ First-generation EGFR TKIs, gefitinib and erlotinib, were designed to combine reversibly with the ATP-binding sites, thus blocking EGFR-induced activation of downstream signaling. The outcome of EGFR 


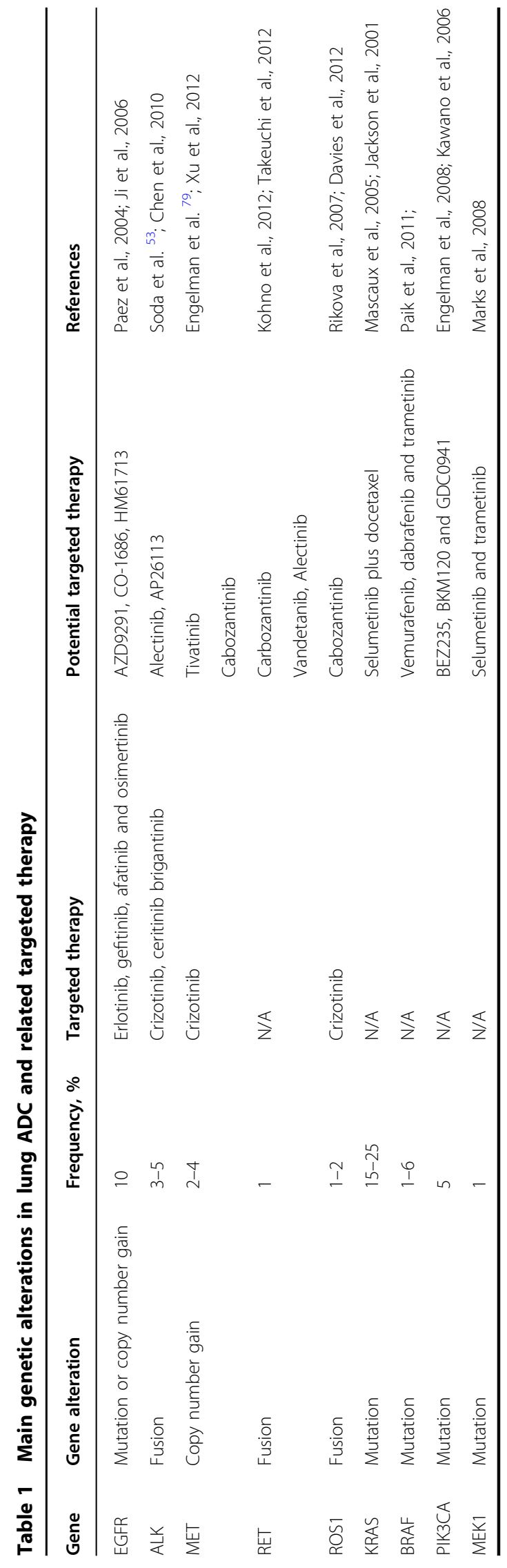

targeting is characterized by the disruption of a number of cellular processes that mirror the physiological consequences of EGFR signal transduction at the level of cell division, angiogenesis and apoptosis ${ }^{32}$. Different randomized controlled phase III trials have demonstrated that first or second generations EGFR TKIs represent the best first-line treatment option in patients with advanced lung ADC and whose tumors harbor EGFR mutations, considerably superior to conventional chemotherapy, because they significantly improved the response rate and progression-free survival (Fig. 1) 1 $^{31,33-35}$.

As mentioned above, like EGFR mutations, ALK rearrangements define a unique molecular subset of NSCLCs. Crizotinib (Xalkori1; Pfizer, CA, USA) is a small-molecule ALK TKI that leads to cell arrest in the G1-S phase, and the induction of apoptosis ${ }^{36}$. Besides EML4-ALK, crizotinib is also active in tumors with c-MET gene amplification and c-ROS kinase mutations ${ }^{21,37}$. Crizotinib has now been approved by the FDA for the treatment of advanced, ALK rearranged NSCLC. The results from two early phase studies demonstrated impressive tumor response rates and prolonged patient survival ${ }^{38}$. Subsequently, several clinical trials, comparing crizotinib to standard chemotherapy in ALK-positive NSCLC patients showed significantly longer progression-free survival in the crizotinib group, leading to full approval of crizotinib by the FDA, EMA, and Japan ${ }^{24,39}$.

The outcome of TK targeting is characterized by the disruption of a number of cellular processes that mirror all levels of the physiological consequences of EGFR signal transduction $^{32}$. Thus, in the context of oncogenic addiction, EGFR TKIs are able to influence the cellular level of apoptosis-related proteins underlying the pro-apoptotic effect of EGFR targeting ${ }^{40,41}$. It has been demonstrated that erlotinib-induced cell growth inhibition is accompanied by G1/S phase arrest, predominantly by the suppression of G1/S-related cyclins and upregulation of the CDK inhibitor $\mathrm{p} 27 \mathrm{KIP}{ }^{42}$. Numerous studies have shown that the acute inactivation of EGFR results in a drastic decline in p-ERK and AKT and a delayed increase in p38 levels. This finally results in a rapid decrease in proliferative stimuli and an increase in pro-apoptotic signals in addicted cancer cells ${ }^{43}$. Orally administered gefitinib or erlotinib are taken up by cancer cells, and reversibly and competitively inhibit the binding of ATP to the phosphate-binding loop. Through the inhibition of ATP binding to EGFR, the EGFR TKIs block autophosphorylation and the activation of downstream signaling pathways, leading to the inhibition of cell proliferation and the induction of apoptosis in cancer cells ${ }^{44}$. Similarly, incubation with crizotinib results in a dosedependent reduction in tumor cell growth, with a clear cell cycle arrest ${ }^{45}$. Inhibition of EML4-ALK with TAE684, a small-molecule ALK inhibitor, or via knockdown using 
RNA interference results in the abrogation of downstream signaling and induction of apoptosis through the activation of the pro-apoptotic protein $\operatorname{Bim}^{46,47}$.

Unfortunately, after TKI treatment, nearly all patients are eventually susceptible to disease progression due to acquired resistance (although resistant clones might have been present before treatment commenced) ${ }^{22}$. The resistant mechanisms identified can be categorized as secondary mutations in EGFR, bypass or alternative activations, or histological transformations ${ }^{23,48,49}$. The gatekeeper Thr790Met mutation is the most frequent secondary EGFR mutation, occurring in $50-65 \%$ of resistant re-biopsies. In addition, HER2 amplifications and mutations have been observed in lung ADC biopsies in 10 and $2 \%$ of tumors with acquired resistance to erlotinib and gefitinib, respectively, but in only $1 \%$ of untreated tumors $^{50}$. Therefore, HER2 may also be responsible for resistance emerging under pressure of treatment, especially with erlotinib ${ }^{51}$. The development of thirdgeneration irreversible inhibitor (AZD9291, osimertinib), which targets both Thr790Met and EGFR TKI-sensitizing mutations, has shown an objective response rate of $61 \%$ and a median progression-free survival of almost 10 months in patients with Thr790 Met-positive NSCLCs who progressed after previous TKI therapy (Fig. 1$)^{52}$.

Secondary mutations in the ALK kinase domain have been identified in approximately 30\% of ALK-positive patients with crizotinib resistance ${ }^{53,54}$. Acquired resistance to crizotonib in these patients inevitably leads to relapse and tumor progression. Moreover, the occurrence of de novo secondary ALK mutations results in variants that are intrinsically less sensitive to the drug ${ }^{55}$. Analysis of pleural fluid from these patients revealed two non-overlapping mutations, L1196M and C1156Y, within the ALK kinase domain. Each independently conferred crizotinib resistance in vitro. The $\mathrm{L} 1196 \mathrm{M}$ substitution is notable because it involves the ALK gatekeeper residue, analogous to T790M in EGFR. The L1196M mutation, which replaces a leucine moiety with a bulkier methionine residue, likely causes resistance by steric interference with crizotinib binding. Since the initial case report of crizotinib resistance, additional second-site ALK mutations have been identified in patient-derived NSCLC specimens ${ }^{54}$.

To overcome these secondary mutations in ALKharboring tumors, several novel ALK inhibitors have recently been developed. Agents, such as ceritinib, alectinib, and lorlatinib (PF-06463922, Pfizer; New York), have several potential advantages over crizotinib including better specificity (e.g., not inhibiting MET and ROS1), greater sensitivity, the ability to cross the blood-brain barrier, and different spectra of activity against resistance mutations to crizotinib ${ }^{31,56,57}$. All of these agents were initially assessed in patients with disease progression on crizotinib and showed substantial activity in this population with a response reported in at least 39\% of patients and median progression-free survival of 5-7 months or more ${ }^{58}$.

Except for secondary mutations, in patients unaffected by TKI treatment wide ranges of resistance mechanisms have been reported. These include increased activity of additional kinases owing to MET, HER2 or ERK amplification, as well as an additional mutation of PIK3CA (which encodes the PI3K p110 $\alpha$ subunit) ${ }^{59}$. Enhanced NF$\mathrm{\kappa B}$ signaling activity has also been proposed as one possible resistance mechanism that is evident from an improved response and survival in patients with EGFR mutations who have an increased expression of the NF- $\kappa B$ inhibitor IкB $\alpha$ (also known as NFKBIA) ${ }^{60}$. In addition, recent data have suggested the pro-apoptotic protein Bim as a biomarker and mediator of TKI-induced apoptosis in EGFR-mutated lung $\mathrm{ADC}^{61}$. The initial mechanism that might explain the different response of wt or mt EGFR lung ADCs to TKIs may include the EGF-mediated autophosphorylation of multiple tyrosine residues linked to the activation of distinct downstream effectors. These effectors may regulate level of Bim and Mcl-1 in mtEGFR cells but not in wtEGFR cells. Thus, the inhibition of EGFR in mtEGFR cells may initiate the apoptotic program via $\mathrm{Bim} / \mathrm{Mcl}-1$ alteration ${ }^{62}$. Moreover, the Bim polymorphism that results in changes in the splicing and deletion of the pro-apoptotic Bcl-2-homology domain (BH3) has been shown potentially to mediate intrinsic resistance to EGFR inhibitors, highlighting the complexity of possible resistance mechanisms (Fig. 1$)^{63}$.

TKI resistance is also associated with epithelialmesenchymal transition (EMT), a process characterized by a loss of polarity and cell-cell contacts in the epithelial cell layers, which undergo a dramatic remodeling of their cytoskeleton $^{64}$. In the context of EMT, upregulation of the receptor protein TK AXL might lead to acquired EGFR TKI resistance in EGFR-mutant NSCLCs (Fig. 1) ${ }^{65}$. In addition, EMT regulated by loss of Mediator Complex Subunit 12 (MED12) has been shown to modulate the response to inhibitors of EGFR, ALK, and BRAF68 through negative regulation of TGF- $\beta$ R2 leading to apoptosis ${ }^{59}$.

As tumors with KRAS mutations do not respond to either gefitinib or erlotinib, it has been suggested that the presence of these mutations be used as a biomarker for predicting resistance of lung ADCs to TKI therapy ${ }^{25}$. However, as described earlier, mutations in EGFR and KRAS are usually mutually exclusive, which makes KRAS an independent therapeutic target ${ }^{66-68}$. Mutations of KRAS are not a chemically druggable target but can potentially be treated with synthetic lethal approaches such as a combination of MEK inhibitors plus PIK3CA or AKT1 inhibitors ${ }^{59}$. For example, in second-line therapy, MEK has been targeted in KRAS-mutated NSCLC tumors by combining the MEK inhibitor selumetinib with 
docetaxel ${ }^{69}$. The suggestion to use MEK inhibitors in clinics was based on their ability to enhance apoptosis in KRAS and mutated NSCLC ${ }^{70}$. Unfortunately, despite the existing rationale for targeting downstream effectors of KRAS such as MEK, clinical trials have failed to confirm the efficacy of this strategy. A possible explanation, which has formed the basis of combined approaches, is an activation of compensatory signaling pathway(s) triggered by the inhibition of MEK, thus providing escape routes for the cancer cells that ensure their survival.

\section{Combination therapies}

Although targeted drugs dramatically improve the outcome of patients with tumors harboring specific alterations, clinical responses are generally short-lived. In most patients with solid tumors, the cancer evolves to become resistant within a few months ${ }^{34,71-73}$. A possible approach to overcoming the limitations of targeted agents is to use combinations rather than monotherapies. For patients with acquired resistance, an option is to continue EGFR TKI therapy in combination with platinum-based doublet chemotherapy. This option is suggested to be beneficial because of the potential tumor heterogeneity at the time of EGFR TKI resistance. Early concurrent combination studies were designed before the discovery of EGFR mutations and the results of these studies in unselected populations showed that combination treatment did not improved survival compared with chemotherapy alone ${ }^{74,75}$. An explanation for this lack of efficacy is that the G1 cell cycle arrest caused by EGFR TKIs might reduce the cell cycle phase-dependent activity of chemotherapy ${ }^{76}$. In contrast, preclinical data have shown that sequential administration of TKIs after conventional chemotherapy might be effective $^{77,78}$. Indeed, co-administration of chemotherapy with TKIs might attenuate the acute effect of EGFR withdrawal because of the effects of chemotherapeutic agents on DNAdamage checkpoints ${ }^{14}$.

As mentioned previously, resistance to TKIs may be developed through different mechanisms including upregulation of bypass signaling pathways. In this case the primary drug target remains unaltered and continues to be inhibited, whereas an alternative kinase becomes activated. There are multiple mechanisms of resistance via bypass pathways, such as MET or HER2 amplifications, and PIK3CA or BRAF mutations ${ }^{23}$. Amplification of MET, the main bypass signaling resistance, has been found in $20 \%$ of EGFR-driven resistant tumors, conferring resistance through ERBB3-mediated activation of downstream PI3K/AKT signaling, and effectively bypassing the inhibited $\mathrm{EGFR}^{79}$. Strategies aimed at co-targeting bypass pathways are being actively pursued in lung $\mathrm{ADC}^{80}$. There are several MET pathway inhibitors in clinical development, including TKIs (crizotinib, cabozantinib, tivantinib, and foretinib) and monoclonal antibodies directed against both MET (onartuzumab) and the HGF ligand (rilotumumab and ficlatuzumab) ${ }^{80}$.

HER2 has been found to be amplified in 12\% of tumors with acquired resistance vs. only $1 \%$ of untreated lung ADCs. When amplified, HER2 is believed to function in parallel with the inhibited EGFR to reactivate common downstream signaling pathways ${ }^{81}$. Dacomitinib (PF00299804, Pfizer; New London, CT, USA) is an irreversible pan-HER inhibitor that has shown remarkable activity in tumors with gefitinib-resistant EGFR T790M or HER2 mutations ${ }^{79}$. Recently, genetic alterations in effectors downstream of EGFR have also been identified as potential mediators of resistance ${ }^{34,81}$.

The ligand-independent activation of RTKs in lung ADC may arise from chromosomal rearrangements related to RTK genes and/or from point mutations or amplification of RTK genes ${ }^{82}$. The involvement of dysregulated RTK-dependent signaling in cellular transformation justifies the rational for the development of RTK inhibitors and their inclusion in targeted cancer therapy. However, the most recent RTK-targeted therapy failed to improve the cure rate because of the activation of defense mechanisms and acquired resistance in tumors ${ }^{83}$. One mechanism that might sustain the drug resistance of tumor cells is autophagy, which is known to be an important catabolic process that regulates the degradation and recycling of organelles and proteins within the cell, maintaining general cellular homeostasis ${ }^{84}$. Numerous scientific reports have highlighted the association between unbalanced regulation of autophagy and cancer ${ }^{85,86}$. Moreover, autophagy stimulation has been associated with resistance of lung ADC to RTK inhibitors, such as erlotinib or gefitinib ${ }^{87}$. Importantly, the degree of autophagy induction by erlotinib has been found to be greater in drug-resistant cells than in sensitive cells, suggesting that its induction may constitute a mechanism of cytoprotection $^{88}$. The disruption of autophagy with chloroquine could accelerate erlotinib-induced apoptosis and overcome resistance of lung ADC cells to treatment with gefitinib or erlotinib. It has been reported that gefitinib causes a strong induction of autophagy in the NSCLC cell line PC- $9^{89}$. The blockage of autophagic flux with clarithromycin was followed by marked induction of cell death $^{90}$. ALK inhibition might also provoke autophagydependent resistance. In the same study, crizotinib, was used to generate resistant LC cell lines and the downregulation of ALK protein was shown to be associated with the induction of autophagy, demonstrating cytoprotective features $^{91}$. Upon inhibition of autophagy with chloroquine, the sensitivity of drug-resistant LC cells to crizotinib was restored, providing an additional rationale for targeting autophagy in the case of resistance to RTK inhibitors $^{87,91}$. The experimental evidence obtained after using MET inhibitors suggests that the cytoprotective 
effect gained by activating autophagy might be a serious obstacle to effective therapy. Therefore, the suppression of autophagy could be an approach to guaranteeing significant improvements in the therapeutic strategy of RTK inhibition $^{92}$.

However, when autophagy is further elevated by treatment in addition to EGFR TKIs, it can induce autophagic cell death. Thus, in erlotinib-resistant HeLaR30 cells, treatment with rapamycin increased autophagic cell death induced by erlotinib. Importantly, in this case cell death was inhibited by knockdown of the autophagy gene $\mathrm{ATG}^{93}$. Similarly, in EGFR TKIresistant LC cells with T790M mutation, the combination of a protein kinase CK2 inhibitor and an EGFR TKI led to a high level of autophagy that degraded EGFR protein and promoted apoptosis ${ }^{94}$. A recent study demonstrated that hypoxia-modulated autophagy was induced by EGFR TKI ${ }^{95}$. Furthermore, the pro-cell survival and pro-cell death roles of autophagy can be switched by adding EGFR TKIs early in hypoxia or by reactivating EGFR later in hypoxia ${ }^{95}$.

Interactions between malignant and neighboring nonmalignant cells create a dynamic tumor microenvironment that can also be therapeutically exploited. Important intercellular communications are driven by a complex and dynamic network of cytokines, chemokines, growth factors, and inflammatory and matrix remodeling enzymes against a background of major perturbations in the physical and chemical properties of lung tumors ${ }^{96}$. Angiogenesis is an essential process in the development, growth and metastasis of NSCLCs. Vascular endothelial growth factor (VEGF) is the major regulator of angiogenesis, and increased expression of VEGF is reportedly associated with poor prognosis ${ }^{57}$. A humanized antibody targeting VEGF, bevacizumab, has shown significant benefit when combined with cytotoxic chemotherapies ${ }^{97,98}$. Moreover, VEGF-targeted therapies exert their effects through a number of potential mechanisms, including inhibition of new vessel growth, regression of newly formed tumor vasculature, alteration of vascular function and tumor blood flow ("normalization"), and direct effects on tumor cells $^{99}$. Because of the presumed cytostatic mechanism of action of anti-angiogenic agents, the efficacy of bevacizumab is most appropriately assessed through survival end points rather than the objective response end points that have traditionally been used with cytotoxic agents. VEGF mediates numerous pro-survival pathways in endothelial cells including induction or activation of Bcl2, Akt, survivin and inhibitor of apoptosis proteins

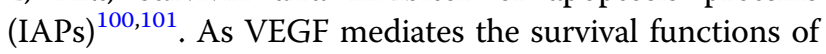
cancer cells, loss of VEGF signaling has been proposed to lead to cancer cell apoptosis ${ }^{102}$.

Recently, a phase II randomized study demonstrated that the addition of bevacizumab to erlotinib led to a significant benefit in terms of median progression-free survival in EGFR-positive NSCLC patients (16 vs. 9.7 months $)^{57}$. Interestingly, the preliminary results of trial have revealed the benefit for patients with EGFRT790M-positive tumors from treatment with the erlotinib plus bevacizumab combination with a median progression-free survival of 16 months compared to 10.5 months for patients without T790M, suggesting a new potential strategy for overcoming T790M-mediated acquired resistance ${ }^{103}$. However, bevacizumab has been shown to increase the response rate with chemotherapy in almost all tumor types studied in phase III trials. For safety reasons, these agents have been restricted to patients with lung ADC with a low risk of hemoptysis ${ }^{104}$. Patients with squamous cell morphology tumors were excluded from the trial because of an increased risk of bleeding events seen after bevacizumab treatment ${ }^{105}$. The FDA and EMA have approved a novel monoclonal antibody ramucirumab directed to the VEGF receptor for use in combination with docetaxel in the second-line treatment of squamous and non-squamous NSCLCs, although modest but statistically significant improvements in overall and median progression-free survival have been observed regardless of histological subtype ${ }^{105}$.

The largest class of anti-VEGF pathway agents comprises the TKIs that inhibit VEGFR. However, these compounds have multiple targets, leading to the variable toxicity and efficacy results seen to date. Nintedanib is an orally administered, small-molecule triple angiokinase inhibitor of VEGF1-3, PDGF- $\alpha$ and $\beta$, and FGFR1-3 which has demonstrated substantial antitumor and antiangiogenic activities in preclinical experiments and in clinical phase I/II trials in patients with NSCLC ${ }^{106,107}$. Nintedanib is the first antiangiogenic agent to demonstrate a survival benefit in the second-line treatment of patients with lung ADCs vs. docetaxel ${ }^{108}$. Further, two independent, multicenter, phase III studies assessed nintedanib combined with either docetaxel or pemetrexed in patients with advanced or recurrent NSCLC for whom first-line chemotherapy had failed. The combination of nintedanib and docetaxel significantly improved median progression-free survival vs. docetaxel alone regardless of histology, whereas overall survival was improved only in patients with lung $\mathrm{ADC}^{108}$. The combination of nintendanib and pemetrexed vs. pemetrexed alone in patients with non-squamous NSCLC significantly improved median progression-free survival but not overall survival ${ }^{109}$.

Sorafenib, a biaryl urea, is an oral small-molecule multikinase inhibitor that is effective against RAF kinase, VEGFR, platelet-derived growth factor receptor (PDGFR), c-KIT, c-RET, and FLT3 kinase ${ }^{110}$. This compound exhibit a significant broad-spectrum dose-dependent antitumor activity against a wide variety of human tumors in preclinical models. In addition to its anti-proliferative 
and anti-angiogenic effects, the anticancer effects of sorafenib are also thought to be mediated by apoptosis induction $^{110}$. Recent studies have suggested that sorafenib-induced apoptosis is associated with downregulation of the antiapoptotic protein Mcl-1 and inhibition of eukaryotic translation initiation factor 4E (eIF4E) phosphorylation ${ }^{111,112}$.

Similar to sorafenib, sunitinib malate has been shown to be a potent inhibitor of VEGF receptors, FLT3, c-KIT, and PDGF receptors in vitro fulfilling its direct antitumor and anti-angiogenic properties ${ }^{113}$. Inhibition of VEGFR and PDGFR by sunitinib prevents further growth of new vessels ${ }^{114}$. Importantly, in other types of cancer one of the mechanisms for the partial resistance to sorafenib and sunitinib has been linked to authophagy development ${ }^{87}$. Moreover, the anticancer effect was restored when these drugs were combined with pharmacological inhibitors of autophagy (chloroquine or bafilomycin A1), which successfully re-activated apoptosis ${ }^{115}$.

Other agents that target VEGF directly, such as vandetanib, and cediranib, have been investigated for the treatment of NSCLC. However, despite promising results in preclinical studies, these compounds have not been found to provide clinical benefit (as measured by the median progression-free rate or overall survival) when combined with pemetrexed as a second-line treatment, compared with placebo, or with erlotinib in previously treated NSCLC patients ${ }^{116,117}$. Moreover, the clinical benefit of TKIs that inhibit VEGFR is limited by toxicity and acquired resistance.

Despite multiple resistance mechanisms and the complexities caused by tumor heterogeneity and microenvironment interactions, chemotherapeutics and molecularly targeted therapies are effective in many disease settings, significantly prolonging patients' lives. The current challenge is to learn from experiences with traditional cytotoxic drugs and the first wave of molecularly targeted agents to use the increasing arsenal of anticancer therapies in the most effective way. Rational drug combinations are often proposed based on in vitro and in vivo synergy between agents. Most importantly, it is essential to stratify patients according to whether they are likely to respond to a particular drug or drug combination.

\section{Immunotherapy}

LC initiation and progression depend not only on the evolving genomics and molecular properties of cancer cells but also on their interaction with the tumor environment, specifically with the immune system ${ }^{118}$. Although NSCLC has historically been considered a nonimmunogenic disease, emerging evidence has demonstrated that the lack of an effective immune response is in fact often the result of specific, active immune-evasive mechanisms, which, if understood, can be overcome therapeutically with significant clinical efficacy. Harnessing this potential has, therefore, become a primary area of clinical interest ${ }^{119,120}$.

The immune system is now recognized as having the potential to destroy cancer cells and inhibit tumor growth through the activation of innate and adaptive respon$\operatorname{ses}^{121}$. Innate immunity mediated by natural killer (NK) cells, polymorphonuclear leukocytes, and mast cells, as well as antigen-presenting cells (APCs), such as macrophages and dendritic cells (DCs), leads to the secretion of interferon gamma (IFN- $\gamma$ ) and perforin, as well as inflammatory cytokines that induce apoptosis of tumor cells. In contrast, adaptive immunity is controlled by $\mathrm{T}$ lymphocytes (CD4+ and $\mathrm{CD}+$ cells) and antibodyproducing $\mathrm{B}$ cells ${ }^{121}$. In this respect, adaptive rather than innate immunity offers the greatest potential for durable, robust anticancer immune responses. Of note, some of the cells involved in innate immunity, such as DCs, macrophages, and NK cells, also play a role in adaptive immunity ${ }^{122}$.

Interaction between the immune system and tumor is based on three phases: elimination, equilibrium and escape. Elimination is a phase of cancer immunoediting in which the innate and adaptive immune systems together detect and eradicate early tumor cells with activated cytotoxic $\mathrm{T}$ cells (CTLs) being major players. The first activating signal is associated with the interaction of $\mathrm{T}$ cell receptors (TCRs) with major histocompatibility complex (MHC) class I molecules on antigen presenting cells (APCs), and provides specificity of response. The second, the so-called "costimulatory signal", stimulates $\mathrm{T}$ cells after conjunction with antigen, and provides molecules on APCs that bind to particular costimulatory receptors on $\mathrm{T}$ cells. The best-known costimulatory molecules are the CD28 family ${ }^{123}$.

In the equilibrium phase the immune system holds the tumor in a state of functional dormancy. The escape phase is characterized by immunoediting insufficiency in restricting tumor growth ${ }^{124}$. As mentioned above, the ability of tumors to escape immunological surveillance is one of the hallmarks of cancer ${ }^{1}$. Entering the immune escape phase tumor cells are able to create an immunosuppressive state within the tumor microenvironment by subverting the same mechanisms that under normal conditions help regulate the immune response and prevent damage of healthy tissues ${ }^{125}$. Key immunosuppressive cell types found in the tumor microenvironment are regulatory $\mathrm{T}$ (Treg) cells, myeloid-derived suppressor cells (MDSCs), and tumor-associated macrophages ${ }^{126}$.

Moreover, specific physiological regulatory mechanisms, or "checkpoints," which play a key role in maintaining normal self-tolerance and limiting the extent of immune responses to infection, can be exploited by tumors as immune resistance mechanisms. Two of the 

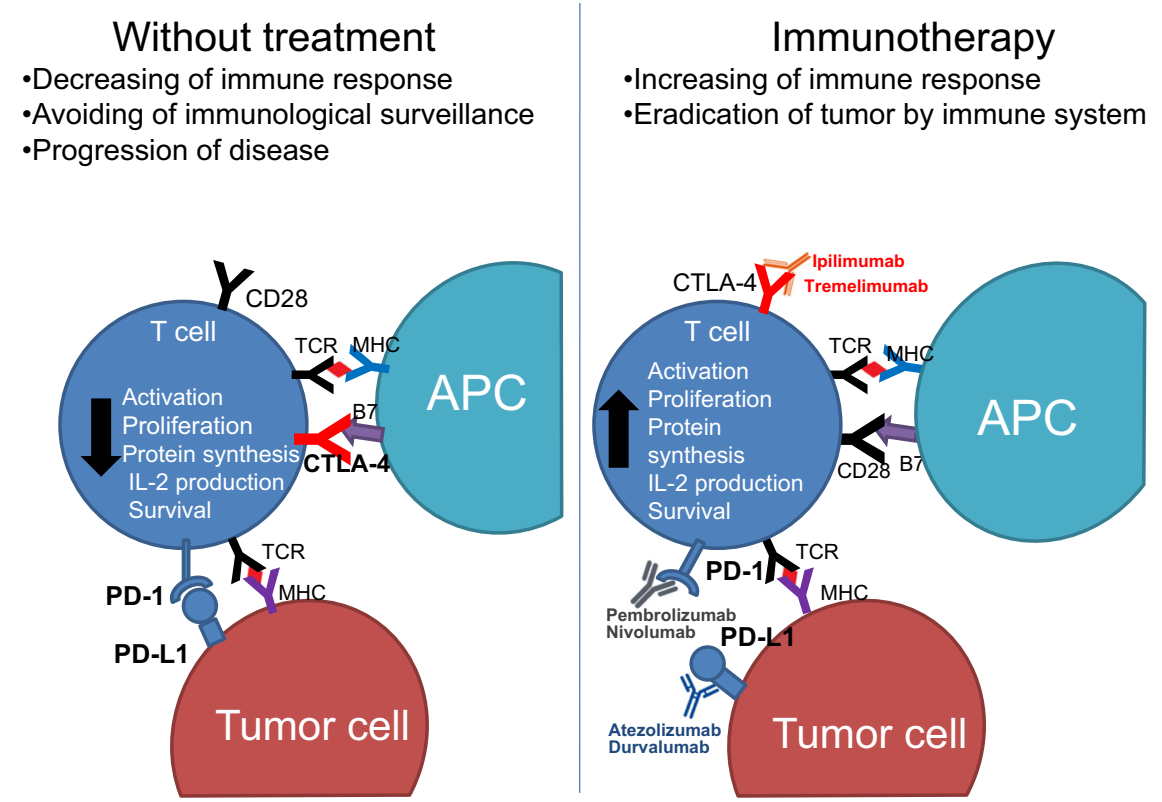

Fig. 2 Link between the immune system state and response of immune checkpoint molecule inhibitors: CTLA-4 inhibitors (ipilimumab and tremelimumab), PD-1 inhibitors (pembrolizumab and nivolumab), PD-L1 inhibitors (atezolizumab and durvalumab). For more detail, see the text

checkpoint receptors most investigated in terms of immunotherapeutic targets for cancer are the cytotoxic $\mathrm{T}$ lymphocyte antigen-4 (CTLA-4) and programmed death1 (PD-1) receptor, which downregulate T-cell activation, proliferation, and function through different mechanisms (Fig. 2) ${ }^{127}$.

CTLA-4 is a protein receptor expressed on the surface of CTLs following their full activation. The binding between CTLA-4 and B7-1 (CD80) or B7-2 (CD86) on APCs prevents the hyperactivity of $\mathrm{T}$ cells under normal conditions. In tumors, $\mathrm{T}$ cells express a high level of CTLA-4, so that, cancer can evade the cytotoxic effect of T cells ${ }^{128}$.

Upregulation of the PD-1 receptor on activated T cells and subsequent binding to one of its ligands, programmed death ligand-1 (PD-L1) or PD-L2, provide an inhibitory signal during the effector phase of the $\mathrm{T}$-cell response, reducing cytokine production, cell proliferation, and cell survival signaling ${ }^{129}$.

Like other tumor types, NSCLC can establish an immunosuppressive tumor microenvironment conducive to tumor growth ${ }^{130,131}$. For instance, NSCLCs have been shown to contain large numbers of Treg cells that constitutively express high level of CTLA-4 on their surface and directly inhibit T-cell proliferation ${ }^{130}$. In addition, in NSCLC, tumor-infiltrating CD8 $+\mathrm{T}$ cells are characterized by increased PD-1 expression associated with impaired immune function. PD-L1 expression has also been found to be upregulated on NSCLC cells, shown correlation with the suppression of the maturation of tumor-infiltrating DCs and reduced tumor T-cell infiltration ${ }^{131}$. Furthermore, overexpression of PD-L1 and PD-L2 has been observed at more advanced disease stage in lung $\mathrm{ADC}^{132}$. Altogether, these data suggest the importance of immune checkpoints for targeting cancer cells. The administration of drugs targeting these molecular pathways may lead to complete or partial eradication of NSCLCs by the immune system.

Indeed, antibody-directed therapies against CTLA-4, PD-1, and PD-L1 have shown remarkable early success in the management of advanced NSCLC (Fig. 2) ${ }^{133}$. Several monoclonal antibodies directed to the PD-1 (nivolumab, pembrolizumab) or its ligand PD-L1 (atezolizumab, durvalumab, avelumab) are in clinical development, and nivolumab and pembroluzimab have been approved by the FDA and EMA for use in patients with advanced NSCLC who have previously been treated with chemotherapy (nivolumab has also been approved in Japan $)^{133}$. Early clinical trials with these agents have shown rapid and durable responses in around 14-20\% of previously treated patients with advanced NSCLC ${ }^{134-136}$. Even though progression-free survival has not been impressive (median 2-4 months), overall survival outcomes are remarkable ${ }^{137}$. Clinical efficacy seems to be independent of histology, but in most of the trials, greater benefit was seen in smokers and in patients with PD-L1- 
positive expression. The toxicity profile of these agents is quite favorable; however, some patients can respond with severe autoimmune disease.

\section{Combined therapy and immunotherapy}

Currently, various combined immunotherapeutic regimens are being investigated in clinical trials for their ability to mediate superior antineoplastic effects compared to monotherapies ${ }^{135}$. Of interest is the combination of PD-1 blockade and CTLA-4 inhibition (nivolumab plus ipilimumab) and combination with other immune checkpoint modulators. In preclinical studies multiple immune checkpoint blockades with combination PD-1 and CTLA4 Ab treatment have been shown to allow increased $\mathrm{T}$-cell responsiveness and decreased T-cell anergy ${ }^{138}$. These approaches have been supported by the results of clinical studies of nivolumab and ipilimumab in patients with NSCLC with squamous and non-squamous morphology ${ }^{139}$. However, a cautious approach is warranted given the potential to exacerbate autoimmunity.

Cytotoxic chemotherapy, targeted therapies and radiotherapy can modulate the immune response of tumors. An understanding of these immunomodulatory effects may enable the design of rational combinations of chemotherapy and immunotherapy. Several phase I/II clinical studies are currently under design to investigate PD-1/ PD-L1 inhibition in combination with chemotherapy for patients with advanced NSCLC. Thus, nivolumab and atezolizumab in combination with chemotherapy as a first-line therapy have also shown promising clinical activity $^{140}$. Cytotoxic chemotherapy with oxaliplatin, gemcitabine or paclitaxel can modulate the immune system through several mechanisms such as inducing immunogenic cell death, a form of cell death that forces DCs to stimulate tumor antigen presentation to $\mathrm{T}$ cells or stimulate $\mathrm{T}$-cell activation via increasing the expression of MHC-1 molecules ${ }^{141}$. Moreover, these cytotoxic agents as well as cyclophosphamide may also stimulate DC maturation and reduce the immunosuppressive function of regulatory $\mathrm{T}$ cells ${ }^{142,143}$.

Combination therapy using PD-1 pathway blockade and EGFR TKIs has also been shown in preclinical studies to be promising. EGFR activation up-regulates the expression of PD-L1 and hence contributes to immune eva$\operatorname{sion}^{144}$. A retrospective study that included 125 patients with NSCLC with mutant and wild-type EGFR, KRAS and ALK, all with PD-L1 expression revealed a correlation between PD-L1 expression and EGFR mutation ${ }^{145}$. Moreover, PD-L1- positive patients had higher sensitivity to EGFR-TKIs than PD-L1-negative patients in terms of the response rate. Interestingly, PD-L1-positive tumors were tightly linked to lung ADC histology ${ }^{145}$. A significant clinical benefit after TKI therapy in PD-L1-positive patients with EGFR-mutant advanced lung ADC was demonstrated $^{146}$.

VEGF may have immunosuppressive effects via the stimulation of MDSCs in peripheral immune organs, promoting regulatory $\mathrm{T}$ cells and inhibiting $\mathrm{DC}$ maturation ${ }^{147}$. As such, VEGF inhibition in combination with a checkpoint inhibitor may have synergistic effects $^{148}$. Several trials are currently assessing different aspects of the combination of antiangiogenic and immunotherapy, including a phase I trial evaluating the safety and tolerability of nivolumab as a maintenance therapy in combination with bevacizumab in NSCLC $^{149}$.

\section{Adoptive immunotherapy}

Other immunotherapeutic approaches including chimeric antigen receptor (CAR) and CD3-based bispecific agents have been associated with systemic cytokine release syndrome $(\mathrm{CRS})^{150}$. CAR is a synthetic molecule designed to redirect $\mathrm{T}$ cells to specific antigens expressed on the surface of tumor cells ${ }^{151}$. CAR T cells can recognize antigens independently of human leukocyte antigen (HLA) unlike to the physiology of T cells and have the ability to affect tumor cells with low HLA expression or with the "wrong" antigen ${ }^{152}$. Tissue factor (TF) or coagulation factor III is overexpressed in many cancer types including $\mathrm{LC}^{153}$. The therapeutic efficacy of TF-CAR $\mathrm{T}$ cells has been estimated in a subcutaneous xenograft model in NOG mice using the human NSCLC cell line NCI-H292 containing the gene encoding luciferase (NCIH292-luc). Intratumoral administration of TF-CAR $\mathrm{T}$ cells demonstrated significant inhibition of the growth of TF-positive NSCLC xenografts in vivo. In addition, TFCAR T cells' ability to suppress TF-positive NSCLC metastasis was revealed in a pulmonary metastasis model of the same mice ${ }^{153}$.

Recently EGFR has been evaluated as potent target for CAR $T$ cell therapy, revealing a correlation between the infusion of CAR-T-EGFR cells and better response in treatment of 11 NSCLC cases ${ }^{154}$. Importantly, the CART-EGFR protocol was safe and feasible for treating EGFRpositive advanced relapsed/refractory NSCLCs, suggesting that CAR $T$ cell therapy could be a promising anticancer strategy for other solid tumors, particularly those with high EGFR expression.

An interesting approach in adoptive immunotherapy for NSCLCs is the use of T lymphocytes targeted to glypican3 (gpc3). In terms of findings, firstly, immunohistochemistry assay showed that gpc3 was expressed in $66.3 \%$ of lung squamous cell cancer samples and in $3.3 \%$ of lung ADC samples but not in normal lung tissues. Second, in two established lung squamous cell cancer xenograft models, CARgpc3 $\mathrm{T}$ cells almost completely eliminated the growth of gpc3-positive cells. The ability of 
CARgpc3 $\mathrm{T}$ cells to persist in vivo and efficiently infiltrate the cancerous tissues was demonstrated. It seems that gpc3 might be a promising target for the treatment of squamous cell $\mathrm{LC}^{155}$.

\section{Conclusions}

Unfortunately, lung ADC is still one of the most aggressive and rapidly fatal tumor types with overall survival less than 5 years. The discovery of oncogenic driver mutations and their role in predicting response to targeted therapies has changed the way in which clinicians approach the diagnosis and treatment of lung ADCs. Although targeted therapies have shown promising results, nearly all patients eventually have disease progression due to acquired resistance. In addition to well-known mechanisms, several novel mechanisms of resistance have recently been discovered, involving new resistance-conferring mutations within the target proteins (such as T790M in EGFR) or activating bypass signal-transduction pathways via unique mutations or changes in the expression level of the key proteins. During the past decade various new strategies aiming to induce cell death in lung ADCs and overcome their broad resistance to treatment, including acquired resistance to targeted therapies, have been developed. One of these is to combine traditional cytotoxic chemotherapy drugs with molecular-targeted compounds, which helps to overcome the limitations of targeted agents. Another approach to combating the resistance of lung ADC includes the combining autophagy inhibitors with RTK inhibitors. Accumulated evidence has revealed great promise in clinical trials using immune therapies for $\mathrm{LC}$, in particular immune checkpoint blockade. The responses to this type of treatment tend to be durable, but still having problem related to reactions from patient to patient. Considerable effort should be made to understand the mechanisms that contribute to durable responses in some patients and lack of response in other patients with the same histological tumor subtype. It has become clear that personalized therapy is coming to the forefront cancer treatment. The selection of the proper therapeutic approach should be based on detailed analysis of histological features, the genetic (mutation) tumor profiles of individual patients, as well as the tumor microenvironment. This information will be essential for better prediction of malignant behavior and improvements in clinical management, which will include combination of targeted and immune therapies.

\footnotetext{
Acknowledgements

This work was supported by Russian Science Foundation (14-25-00056). The work in the authors' laboratories is being supported by Russian President Fund (NSH-7082.2016), Russian Fund for Basic Research (16-04-00527) as well as the Stockholm (161292) and Swedish (160733) Cancer Societies, the Swedish Childhood Cancer Foundation (PR2016-0090), the Swedish Research Council (521-2014-2258).
}

\section{Competing interests}

The authors declare that they have no competing interests.

Publisher's note: Springer Nature remains neutral with regard to jurisdictional claims in published maps and institutional affiliations.

Received: 27 July 2017 Revised: 18 August 2017 Accepted: 13 September 2017

Published online: 25 January 2018

\section{References}

1. Hanahan, D. \& Weinberg, R. A. Hallmarks of cancer: the next generation. Cell 144, 646-674 (2011).

2. Hanahan, D. \& Weinberg, R. A. The hallmarks of cancer. Cell 100, 57-70 (2000).

3. Stewart B. W. and Wild C. P. World Cancer Report 2014 (Lyon, France: International Agency for Research on Cancer, 2015).

4. Sher, T., Dy, G. K. \& Adjei, A. A. Small cell lung cancer. Mayo. Clin. Proc. 83, 355-367 (2008).

5. Noguchi, M., Morikawa, A. \& Kawasaki, M. et al. Small adenocarcinoma of the lung. Histologic characteristics and prognosis. Cancer 75, 2844-2852 (1995).

6. Zappa, C. \& Mousa, S. A. Non-small cell lung cancer: current treatment and future advances. Transl. Lung Cancer Res. 5, 288-300 (2016).

7. Brambilla, E. et al. Large cell carcinoma. In: W. Travis, E. Brambilla, H. MüllerHermelink, C. C. Harris (eds). World Health Organization Classification of Tumours Pathology and Genetics of Tumours of the Lung, Pleura, Thymus and Heart 45-50. (WHO Press, Geneva, 2004).

8. Viktorsson, K., Lewensohn, R. \& Zhivotovsky, B. Systems biology approaches to develop innovative strategies for lung cancer therapy. Cell Death Dis. 29, e1260 (2014).

9. Travis, W. D. et al. International association for the study of lung cancer/ american thoracic society/european respiratory society international multidisciplinary classification of lung adenocarcinoma. J. Thorac. Oncol. 6, 244-285 (2011).

10. Pao, W. \& Girard, N. New driver mutations in non-small-cell lung cancer. Lancet Oncol. 12, 175-180 (2011).

11. Kris, M. G. et al. Using multiplexed assays of oncogenic drivers in lung cancers to select targeted drugs. JAMA. 311, 1998-2006 (2014).

12. Weinstein, I. B. Cancer. Addiction to oncogenes - the Achilles' heel of cancer. Science 297, 63-64 (2001).

13. Fisher, G. H. et al. Induction and apoptotic regression of lung adenocarcinomas by regulation of a K-Ras transgene in the presence and absence of tumor suppressor genes. Genes Dev. 15, 3249-3262 (2001).

14. Stella, G. M., Luisetti, M., Pozzi, E. \& Comoglio, P. M. Oncogenes in non-smallcell lung cancer: emerging connections and novel therapeutic dynamics. Lancet Respir. Med. 1, 251-261 (2013).

15. Sharma, S. V., Bell, D. W., Settleman, J. \& Haber, D. A. Epidermal growth factor receptor mutations in lung cancer. Nat. Rev. Cancer 7, 169-181 (2007).

16. Sharma, S. V., Gajowniczek, P. \& Way, I. P. et al. A common signaling cascade may underlie "addiction" to the Src, BCR-ABL, and EGF receptor oncogenes. Cancer Cell 10, 425-435 (2006).

17. Scheff, R. J. \& Schneider, B. J. Non-small-cell lung cancer: treatment of late stage disease: chemotherapeutics and new frontiers. Semin. Intervent. Radiol. 30, 191-198 (2013).

18. Ciardiello, F. \& Tortora, G. EGFR antagonists in cancer treatment. N. Engl. J. Med. 358, 1160-1174 (2008).

19. Huang, L. \& Fu, L. Mechanisms of resistance to EGFR tyrosine kinase inhibitors. Acta Pharm. Sin B 5, 390-401 (2015).

20. Gazdar, A. F. Activating and resistance mutations of EGFR in non-small-cell lung cancer: role in clinical response to EGFR tyrosine kinase inhibitors. Oncogene. 28, 24-31 (2009).

21. Yasuda, H. et al. Structural, biochemical, and clinical characterization of epidermal growth factor receptor (EGFR) exon 20 insertion mutations in lung cancer. Sci. Transl. Med. 5, 177-216 (2013).

22. Shtivelman, E., Beer, T. M. \& Evans, C. P. Molecular pathways and targets in prostate cancer. Oncotarget 5, 7217-7259 (2014).

23. Gainor, J. F. \& Shaw, A. T. Emerging paradigms in the development of resistance to tyrosine kinase inhibitors in lung cancer. J. Clin. Oncol. $\mathbf{3 1}$ 3987-3996 (2013). 
24. Alamgeer, M., Ganju, V. \& Watkins, D. N. Novel therapeutic targets in nonsmall cell lung cancer. Curr. Opin. Pharmacol. 13, 394-401 (2013).

25. Massarelli, E. et al. KRAS mutation is an important predictor of resistance to therapy with epidermal growth factor receptor tyrosine kinase inhibitors in non-small-cell lung cancer. Clin. Cancer Res. 13, 2890-2896 (2007).

26. Riely, G. J., Marks, J. \& Pao, W. KRAS mutations in non-small cell lung cancer. Proc. Am. Thorac. Soc. 6, 201-205 (2009)

27. Sadiq, A. A. \& Salgia, R. MET as a possible target for non-small-cell lung cancer. J. Clin. Oncol. 31, 1089-1096 (2013).

28. Minguet, J., Smith, K. H. \& Bramlage, P. Targeted therapies for treatment of non-small cell lung cancer--Recent advances and future perspectives. Int. J. Cancer 138, 2549-2561 (2016).

29. Pikor, L. A., Ramnarine, V. R., Lam, S. \& Lam, W. L. Genetic alterations defining NSCLC subtypes and their therapeutic implications. Lung Cancer 82, 179-189 (2013).

30. Inoue, A. et al. First-line gefitinib for patients with advanced non-small-cell lung cancer harboring epidermal growth factor receptor mutations without indication for chemotherapy. J. Clin. Oncol. 27, 1394-1400 (2009).

31. Mok, T. S. et al. Gefitinib or carboplatin-paclitaxel in pulmonary adenocarcinoma. N. Engl. J. Med. 361, 947-957 (2009).

32. Castillo, L. et al. Pharmacological background of EGFR targeting. Ann. Oncol. 15, 1007-1012 (2004)

33. Maemondo, M. et al. Gefitinib or chemotherapy for non-small-cell lung cancer with mutated EGFR. N. Engl. J. Med. 362, 2380-2388 (2010).

34. Sequist, L. V. et al. Phase III Study of Afatinib or Cisplatin Plus Pemetrexed in Patients With Metastatic Lung Adenocarcinoma With EGFR Mutations. J. Clin. Oncol. 31, 3327-3334 (2013).

35. Yang, J. C. et al. Afatinib for patients with lung adenocarcinoma and epidermal growth factor receptor mutations (LUX-Lung 2): a phase 2 trial. Lancet Oncol. 13, 539-548 (2012)

36. Christensen, J. G. et al. Cytoreductive antitumor activity of PF-2341066, a novel inhibitor of anaplastic lymphoma kinase and c-Met, in experimental models of anaplastic large-cell lymphoma. Mol. Cancer Ther. 6, 3314-3322 (2007)

37. Zou, H. Y. et al. An orally available small-molecule inhibitor of c-Met, PF2341066, exhibits cytoreductive antitumor efficacy through antiproliferative and antiangiogenic mechanisms. Cancer Res. 67, 4408-4417 (2007)

38. Kwak, E. L. et al. Anaplastic lymphoma kinase inhibition in non-small-cell lung cancer. N. Engl. J. Med. 363, 1693-1703 (2010).

39. Camidge, D. R. et al. Activity and safety of crizotinib in patients with ALK positive non-small-cell lung cancer: updated results from a phase 1 study. Lancet Oncol. 13, 1011-1019 (2012).

40. Ciardiello, F. et al. Antiangiogenic and antitumor activity of anti-epidermal growth factor receptor C225 monoclonal antibody in combination with vascular endothelial growth factor antisense oligonucleotide in human GEO colon cancer cells. Clin. Cancer Res. 6, 3739-3747 (2000).

41. Baselga, J. The EGFR as a target for anticancer therapy-focus on cetuximab. Eur. J. Cancer 37, 16-22 (2001)

42. Bronte, G. et al. Are erlotinib and gefitinib interchangeable, opposite or complementary for non-small cell lung cancer treatment? Biological, pharmacological and clinical aspects. Crit. Rev. Oncol. Hematol. 89, 300-313 (2014).

43. Costa, D. B. et al. BIM mediates EGFR tyrosine kinase inhibitor-induced apoptosis in lung cancers with oncogenic EGFR mutations. PLoS Med. 4 1669-1679 (2007).

44. Tang, J., Salama, R., Gadgeel, S. M., Sarkar, F. H. \& Ahmad, A. Erlotinib resistance in lung cancer: current progress and future perspectives. Front. Pharmacol. 4, 15 (2013)

45. Megiorni, F. et al. Crizotinib-induced antitumour activity in human alveolar rhabdomyosarcoma cells is not solely dependent on ALK and MET inhibition. J. Exp. Clin. Cancer Res. 34, e112 (2015).

46. Takezawa, K., Okamoto, I., Nishio, K., Jänne, P. A. \& Nakagawa, K. Role of ERKBIM and STAT3-survivin signaling pathways in ALK inhibitor-induced apoptosis in EML4-ALK positive lung cancer. Clin. Cancer Res. 17, 2140-2148 (2011).

47. Katayama, R. et al. Mechanisms of acquired crizotinib resistance in ALKrearranged lung cancers. Sci. Transl. Med. 4, 120-137 (2012).

48. Gainor, J. F. \& Shaw, A. T. Novel targets in non-small cell lung cancer: ROS1 and RET fusions. Oncologist 18, 865-875 (2013).

49. Niederst, M. J. \& Engelman, J. A. Bypass mechanisms of resistance to receptor tyrosine kinase inhibition in lung cancer. Sci. Signal. 6, 294-310 (2013).
50. Mazieres, J. et al. Lung cancer that harbors an HER2 mutation: epidemiologic characteristics and therapeutic perspectives. J. Clin. Oncol. 31, 1997-2003 (2013)

51. Takezawa, K et al. HER2 amplification: a potential mechanism of acquired resistance to EGFR inhibitionin EGFR-mutant lung cancers that lack the second-site EGFRT790M mutation. Cancer Discov. 2, 922-933 (2012).

52. Jänne, P. A. et al. AZD9291 in EGFR inhibitor-resistant non-small-cell lung cancer. N. Engl. J. Med. 372, 1689-1699 (2015).

53. Soda, $M$. et al. Identification of the transforming EML4-ALK fusion gene in non-small-cell lung cancer. Nature. 448, 561-566 (2007).

54. Shaw, A. T. et al. Clinical features and outcome of patients with non-small-cell lung cancer whoharbor EML4-ALK. J. Clin. Oncol. 27, 4247-4253 (2009).

55. Voena, C. \& Chiarle, R. The battle against ALK resistance: successes and setbacks. Expert Opin. Investig. Drugs. 21, 1751-1754 (2012).

56. Friboulet, L. et al. The ALK inhibitor ceritinib overcomes crizotinib resistance in non-small cell lung cancer. Cancer Discov 4, 662-673 (2014).

57. Seto, T. et al. Erlotinib alone or with bevacizumab as first-line therapy in patients with advanced non-squamous non-small-cell lung cancer harboring EGFR mutations (JO25567): an open-label, randomised, multicentre, phase 2 study. Lancet. Oncol. 15, 1236-1244 (2014).

58. Kodama, T., Tsukaguchi, T., Yoshida, M., Kondoh, O. \& Sakamoto, H. Selective ALK inhibitor alectinib with potent antitumor activity in models of crizotinib resistance. Cancer. Lett. 351, 215-221 (2014).

59. Rosell, R., Bivona, T. G. \& Karachaliou, N. Genetics and biomarkers in personalisation of lung cancer treatment. Lancet. 382, 720-731 (2013).

60. Bivona, T. G. et al. FAS and NF-KB signalling modulate dependence of lung cancers on mutant EGFR. Nature. 471, 523-526 (2011).

61. Nakagawa, T. et al. EGFR-TKI resistance due to BIM polymorphism can be circumvented in combination with HDAC inhibition. Cancer. Res. 73, 2428-2434 (2013).

62. Yamaguchi, $H$. et al. Caspase-independent cell death is involved in the negative effect of EGF receptor inhibitors on cisplatin in non-small cell lung cancer cells. Clin. Cancer. Res. 19, 845-854 (2013).

63. Spraggs, C. F. et al. Different effects of the BIM deletion polymorphism on treatment of solid tumors by the tyrosine kinase inhibitors (TKI) pazopanib, sunitinib, and lapatinib. Ann. Oncol. 26, 1515-1517 (2015).

64. Thiery, J. P. Epithelial-mesenchymal transitions in development and pathologies. Curr. Opin. Cell. Biol. 15, 740-746 (2003).

65. Zhang, Z. et al. Activation of the AXL kinase causes resistance to EGFRtargeted therapy in lung cancer. Nat. Genet. 44, 852-860 (2012).

66. Kosaka, T. et al. Analysis of epidermal growth factor receptor gene mutation in patients with non-small cell lung cancer and acquired resistance to gefitinib. Clin. Cancer Res. 12, 5764-5769 (2006).

67. Schmid, K. et al. EGFR/KRAS/BRAF mutations in primary lung adenocarcinomas and corresponding locoregional lymph node metastases. Clin. Cancer Res. 15, 4554-4560 (2009)

68. Kris, M. G. et al. Identification of driver mutations in tumor specimens from 1,000 patients with lung adenocarcinoma: the NCl's Lung Cancer Mutation Consortium (LCMC). J. Clin. Oncol. 29l, e7506 (2011).

69. Janne, P. A., Mann, H. \& Ghiorghiu, D. Study Design and Rationale for a Randomized, Placebo-Controlled, Double-Blind Study to Assess the Efficacy and Safety of Selumetinib in combination With Docetaxel as Second-Line Treatment in Patients With KRAS-Mutant Advanced Non-Small Cell Lung Cancer (SELECT-1). Clin. Lung. Cancer 17, 1-4 (2016).

70. MacKeigan, J. P., Collins, T. S. \& Ting, J. P. MEK inhibition enhances paclitaxelinduced tumor apoptosis. J. Biol. Chem. 275, 38953-38956 (2000).

71. Sequist, L. V. et al. Genotypic and histological evolution of lungcancers acquiring resistance to EGFR inhibitors. Sci. Transl. Med. 3, 75-96 (2011)

72. Sechler, M. et al. Non-small-cell lung cancer: molecular targeted therapy and personalized medicine - drug resistance, mechanisms, and strategies. Pharmgenomics Pers. Med. 6, 25-36 (2013).

73. Tartarone, A. et al. Mechanisms of resistance to EGFR tyrosine kinase inhibitors gefitinib/ erlotinib and to ALK inhibitor crizotinib. Lung Cancer $\mathbf{8 1}$ 328-336 (2013)

74. Giaccone, G. et al. Epidermal growth factor receptor expression analysis in chemotherapy-naive patients with advanced non-small-cell lung cancer treated with gefitinib or placebo in combination with platinum-based chemotherapy. J. Cancer. Res. Clin. Oncol. 135 467-476 (2009) 
75. Herbst, R. S. et al. TRIBUTE: a phase III trial of erlotinib hydrochloride (OSI-774) combined with carboplatin and paclitaxel chemotherapy in advanced nonsmall-cell lung cancer. J. Clin. Oncol. 23, 5892-5899 (2005).

76. Gumerlock, P., Pryde, B. J. \& Kimura, T. Enhanced cytotoxicity of docetaxel OSI-774 combination in non-small cell lung carcinoma. Proc. Am. Soc. Clin. Oncol. 22, 662 (2003). (abstr 2661).

77. Li, T., Ling, Y.-H., Goldman, I. D. \& Perez-Soler, R. Schedule-dependent cytotoxic synergism of pemetrexed and erlotinib in human non-small cell lung cancer cells. Clin. Cancer. Res. 13, 3413-3422 (2007).

78. Cheng, $\mathrm{H}$. et al. In vitro sequence-dependent synergism between paclitaxel and gefitinib in human lung cancer cell lines. Cancer. Chemother. Pharmacol. 67, 637-646 (2011)

79. Engelman, J. A. et al. MET amplification leads to gefitinib resistance in lung cancer by activating ERBB3 signaling. Science 316, 1039-1043 (2007).

80. Lovly, C. M. \& Shaw, A. T. Molecular pathways: resistance to kinase inhibitors and implications for therapeutic strategies. Clin. Cancer. Res. 20, 2249-2256 (2014)

81. Ohashi, $\mathrm{K}$. et al. Lung cancers with acquired resistance to EGFR inhibitors occasionally harbor BRAF gene mutations but lack mutations in KRAS, NRAS, or MEK1. Proc. Natl Acad. Sci. USA 109, 2127-2133 (2012).

82. Shaw, A. T., Hsu, P. P., Awad, M. M. \& Engelman, J. A. Tyrosine kinase gene rearrangements in epithelial malignancies. Nat. Rev. Cancer 13, 772-787 (2013)

83. Jackman, D. et al. Clinical definition of acquired resistance to epidermal growth factor receptor tyrosine kinase inhibitors in non-small-cell lung cancer. J. Clin. Oncol. 28, 357-360 (2010).

84. Mizushima, N. Autophagy: process and function. Genes Dev. 21, 2861-2873 (2007)

85. Corcelle, E. A., Puustinen, P. \& Jäättelä, M. Apoptosis and autophagy: targeting autophagy signalling in cancer cells_-'trick or treats'? FEBS J. 276, 6084-6096 (2009)

86. Eisenberg-Lerner, A. \& Kimchi, A. The paradox of autophagy and its implication in cancer etiology and therapy. Apoptosis. 14, 376-391 (2009).

87. Aveic, S. \& Tonini, G. P. Resistance to receptor tyrosine kinase inhibitors in solid tumors: can we improve the cancer fighting strategy by blocking autophagy? Cancer Cell Int. 16, 62-70 (2016).

88. Zou, Y. et al. The autophagy inhibitor chloroquine overcomes the innate resistance of wild-type EGFR non-small-cell lung cancer cells to erlotinib. J. Thorac. Oncol. 8, 693-702 (2013).

89. Sugita, S. et al. EGFR-independent autophagy induction with gefitinib and enhancement of its cytotoxic effect by targeting autophagy with clarithromycin in non-small cell lung cancer cells. Biochem. Biophys. Res. Commun. 461, 28-34 (2015)

90. Nakamura, M., Kikukawa, Y., Takeya, M., Mitsuya, H. \& Hata, H. Clarithromycin attenuates autophagy in myeloma cells. Int. J. Oncol. 37, 815-820 (2010).

91. Ji, M. et al. PD-1/PD-L1 expression in non-small-cell lung cancer and its correlation with EGFR/KRAS mutations. Cancer. Biol. Ther. 17, 407-413 (2016).

92. Humbert, M. et al. Protective autophagy is involved in resistance towards MET inhibitors in human gastric adenocarcinoma cells. Biochem. Biophys. Res. Commun. 431, 264-269 (2013).

93. Fung, C., Chen, X., Grandis, J. R. \& Duvvuri, U. EGFR tyrosine kinase inhibition induces autophagy in cancer cells. Cancer. Biol. Ther. 13, 1417-1424 (2012).

94. So, K. S. et al. Autophagosome-mediated EGFR downregulation induced by the CK2 inhibitor enhances the efficacy of EGFR-TKI on EGFR-mutant lung cancer cells with resistance by T790M. PLOS ONE 9, e114000 (2014).

95. Chen, Y. J. et al. Lapatinib induces autophagic cell death and differentiation in acute myeloblastic leukemia. Oncol. Targets Ther. 9, 4453-4464 (2016).

96. Tsao, A. S., Scagliotti, G. V. \& Bunn, P. A. Jr et al. Scientific advances in lung cancer 2015. J. Thorac. Oncol. 11, 613-638 (2016).

97. Sandler, A., Gray, R. \& Perry, M. C. et al. Paclitaxel-carboplatin alone or with bevacizumab for non-small-cell lung cancer. N. Engl. J. Med. 355, 2542-2550 (2006)

98. Reck, M., von Pawel, J. \& Zatlouka, P. et al. First-line Bevacizumab combined with Cisplatin/Gencitabine (CG) in patients (pts) with advanced or recurrent non-squamous, non-small cell lung cancer (NSCLC): AVAlL (BO17704), a phase III randomized study. J. Thorac. Oncol. 12, 1487-1488 (2008).

99. Ellis, L. M., Rosen, L. \& Gordon, M. S. Overview of anti-VEGF therapy and angiogenesis. Part 1: Angiogenesis inhibition in solid tumor malignancies. Clin. Adv. Hematol. Oncol. 4, 11-20 (2006).
100. Fujio, Y. \& Walsh, K. Akt mediates cytoprotection of endothelial cells by vascular endothelial growth factor in an anchorage-dependent manner J. Biol. Chem. 274, 16349-1654 (1999).

101. Gerber, H. P. et al. Vascular endothelial growth factor regulates endothelia cell survival through the phosphatidylinositol 3'-kinase/Akt signal transduction pathway. Requirement for Flk-1/KDR activation. J. Biol. Chem. 273 30336-30343 (1998).

102. Niu, G. \& Chen, X. Vascular endothelial growth factor as an anti-angiogenic target for cancer therapy. Curr. Drug Targets 11, 1000-1017 (2010).

103. Stahel, R. et al. 3BA: a phase II trial of erlotinib (E) and bevacizumab (B) in patients with advanced non-small-cell lung cancer (NSCLC) with activating epidermal growth factor receptor (EGFR) mutations with and without T790M mutation. The Spanish Lung Cancer Group (SLCG) and the European Thoracic Oncology Platform (ETOP) BELIEF trial. Eur. J. Cancer 51, 711-720 (2015).

104. Tabchi, S. \& Blais, N. Antiangiogenesis for advanced non-small-cell lung cancer in the era of immunotherapy and personalized medicine. Front. Oncol. 7, 52 (2017).

105. Clément-Duchêne, C. \& Wakelee, H. Antiangiogenic agents and vascular disrupting agents for the treatment of lung cancer: a review. J. Thorac. Oncol. 5. 129-139 (2010).

106. Hilberg, F. et al. BIBF 1120: triple angiokinase inhibitor with sustained receptor blockade and good antitumor efficacy. Cancer Res. 68, 4774-4782 (2008).

107. Kutluk Cenik, B., Ostapoff, K. T., Gerber, D. E. \& Brekken, R. A. BIBF 1120 (nintedanib), a triple angiokinase inhibitor, induces hypoxia but not EMT and blocks progression of preclinical models of lung and pancreatic cancer. Mol. Cancer. Ther. 12, 992-1001 (2013).

108. Reck, M. et al. A phase II double-blind study to investigate efficacy and safety of two doses of the triple angiokinase inhibitor BIBF 1120 in patients with relapsed advanced non-small-cell lung cancer. Ann. Oncol. 22, 1374-1381 (2011)

109. Hanna, N. H. et al. Nintedanib plus pemetrexed versus placebo plus pemetrexed in patients with relapsed or refractory, advanced non-small cell lung cancer (LUME-Lung 2): A randomized, double-blind, phase III trial. Lung Cancer 102, 65-73 (2016)

110. Wilhelm, S. M., Carter, C. \& Tang, L. et al. BAY 43-9006 exhibits broad spectrum oral antitumor activity and targets theRAF/MEK/ERK pathway and receptor tyrosine kinases involved in tumor progression and angiogenesis. Cancer. Res. 64, 7099-7109 (2004).

111. Yu, C. et al. The role of $\mathrm{Mcl}-1$ downregulation in the proapoptotic activity of the multikinase inhibitor BAY 43-9006. Oncogene 24, 6861-6869 (2005).

112. Dudgeon, $\mathrm{C}$. et al. Inhibiting oncogenic signaling by sorafenib activates PUMA via GSK3 $\beta$ and NF-kB to suppress tumor cell growth. Oncogene $\mathbf{3 1}$ 4848-4858 (2012).

113. Faivre, S., Djelloul, S. \& Raymond, E. New paradigms in anticancer therapy: targeting multiplesignaling pathways with kinase inhibitors. Semin. Oncol. 33 407-420 (2006).

114. Ikezoe, T. et al. The antitumor effects ofsunitinib (formerly SU11248) against a variety of human hematologic malignancies: enhancement of growth inhibition via inhibition of mammalian target of rapamycin signaling. Mol. Cancer. Ther. 5, 2522-2530 (2006).

115. Yuan, $\mathrm{H}$. et al. Inhibition of autophagy significantly enhances combination therapy with sorafenib and HDAC inhibitors for human hepatoma cells. World J. Gastroenterol. 20, 4953-4962 (2014).

116. Goss, G. D. et al. Randomized, double-blind trial of carboplatin and paclitaxel with either daily oral cediranib or placebo in advanced non-small-cell lung cancer: NCIC clinical trials group BR24 study. J. Clin. Oncol. 28, 49-55 (2010)

117. de Boer, R. H. et al. Vandetanib plus pemetrexed for the second-line treatment of advanced non-small-cell lung cancer: a randomized, double-blind phase III trial. J. Clin. Oncol. 29, 1067-1074 (2011).

118. Forde, P. M., Kelly, R. J. \& Brahmer, J. R. New strategies in lung cancer: translating immunotherapy into clinical practice. Clin. Cancer. Res. 20 1067-1073 (2014).

119. Shepherd, F. A., Douillard, J. Y. \& Blumenschein, G. R. Jr. Immunotherapy for non-small cell lung cancer: Novel approaches to improve patient outcome. J. Thorac. Oncol. 6, 1763-1773 (2011).

120. Brahmer, J. R. Harnessing the immune system for the treatment of non-smallcell lung cancer. J. Clin. Oncol. 31, 1021-1028 (2013).

121. Vesely, M. D., Kershaw, M. H., Schreiber, R. D. \& Smyth, M. J. Natural innate and adaptive immunity to cancer. Annu. Rev. Immunol. 29, 235-271 (2011). 
122. Liu, Y. \& Zeng, G. Cancer and innate immune system interactions:Translational potentials for cancer immunotherapy. J. Immunother. 35, 299-308 (2012).

123. Alarcón, B., Mestre, D. \& Martínez-Martín, N. The immunological synapse: a cause or consequence of T-cell receptor triggering? Immunology. 133 420-425 (2011)

124. Mittal, D., Gubin, M. M., Schreiber, R. D. \& Smyth, M. J. New insights into cancer immunoediting and its three component phases--elimination, equilibrium and escape. Curr. Opin. Immunol. 27, 16-25 (2014).

125. Schreiber, R. D., Old, L. J. \& Smyth, M. J. Cancer immunoediting: Integrating immunity's roles in cancer suppression and promotion. Science $\mathbf{3 3 1}$ 1565-1570 (2011).

126. Mellman, I., Coukos, G. \& Dranoff, G. Cancer immunotherapy comes of age. Nature. 480, 480-489 (2011)

127. Pardoll, D. M. The blockade of immune checkpoints in cancer immunotherapy. Nat. Rev. Cancer 12, 252-264 (2012).

128. Aldarouish, M. \& Wang, C. Trends and advances in tumor immunology and lung cancer immunotherapy. J. Exp. Clin. Cancer. Res. 35, 157 (2016).

129. Carbone, D. P., Gandara, D. R., Antonia, S. J., Zielinski, C. \& Paz-Ares, L. NonSmall-Cell Lung Cancer: Role of the Immune System and Potential for Immunotherapy. J. Thorac. Oncol. 10, 974-984 (2015).

130. Woo, E. Y. et al. Regulatory CD4(+)CD25(+) T cells in tumors from patients with early-stage non-small cell lung cancer and late-stage ovarian cancer. Cancer Res. 61, 4766-4772 (2001).

131. Chen, Y. B., Mu, C. Y. \& Huang, J. A. Clinical significance of programmed death-1 ligand-1 expression in patients with non-small cell lung cancer: A 5year-follow-up study. Tumori. 98, 751-755 (2012).

132. Zhang, $Y$. et al. Protein expression of programmed death 1 ligand 1 and ligand 2 independently predict poor prognosis in surgically resected lung adenocarcinoma. Onco Targets Ther. 7, 567-573 (2014).

133. Topalian, S. L., Weiner, G. J. \& Pardoll, D. M. Cancer immunotherapy comes of age. J. Clin. Oncol. 29, 4828-4836 (2011).

134. Gettinger, S. et al. Nivolumab monotherapy for first-line treatment of advanced non-small-cell lung cancer. J. Clin. Oncol. 34, 2980-2987 (2016).

135. Rizvi, N. A. et al. Activity and safety of nivolumab, an anti-PD-1 immune check point inhibitor, for patients with advanced, refractory squamous non-smallcell lung cancer (CheckMate 063): A phase 2, single-arm trial. Lancet Oncol. 16, 257-265 (2015).

136. Fehrenbacher, L. et al Atezolizumab versus docetaxel for patients with previously treated non-small-cell lung cancer (POPLAR): a multicentre, openlabel, phase 2 randomised controlled trial. Lancet. 387, 1837-1846 (2016).

137. Hirsch, F. R. et al. Lung cancer: current therapies and new targeted treatments. Lancet. 389, 299-311 (2017).

138. Curran, M., Montalvo, W., Yagita, H. \& Allison, J. PD-1 and CTLA-4 combination blockade expands infiltrating $T$ cells and reduces regulatory $T$ and myeloid cells within B16 melanoma tumors. Proc. Natl Acad. Sci. USA 107, 4275-4280 (2010)
139. Herbst, R. et al Pembrolizumab versus docetaxel for previously treated, PDL1-positive, advanced non-small-cell lungcancer (KEYNOTE-010): a randomised controlled trial. Lancet. 387, 1540-1550 (2015).

140. Antonia, S. J., Larkin, J. \& Ascierto, P. A. Imuno-oncology combinations: a review of clinical experience and future prospects. Clin. Cancer Res. 20 6258-6268 (2014)

141. Kroemer, G., Galluzzi, L., Kepp, O. \& Zitvogel, L. Immunogenic cell death in cancer therapy. Annu. Rev. Immunol. 31, 51-72 (2013).

142. Kodumudi, K. et al. A novel chemoimmunomodulating property of docetaxel: suppression of myeloid-derived suppressor cells in tumor bearers. Clin. Cancer Res. 16, 4583-4594 (2010).

143. Ghiringhelli, F. et al. Metronomic cyclophosphamide regimen selectively depletes CD4+CD25+ regulatory $T$ cells and restores $T$ and NK effector functions in end stage cancer patients. Cancer Immunol. Immunother. 56, 641-648 (2007)

144. Akbay, E. et al. Activation of the PD-1 pathway contributes to immune escape in EGFR-driven lung tumors. Cancer Discov. 3, 1355-1363 (2013).

145. D'Incecco, A. et al. PD-1 and PD-L1 expression in molecularly selected nonsmall-cell lung cancer patients. Br. J. Cancer. 112, 95-102 (2015).

146. Lin, K., Cheng, J., Yang, T., Li, Y. \& Zhu, B. EGFR-TKI down-regulates PDL1 in EGFR mutant NSCLC through inhibiting NF-kappaB. Biochem. Biophys. Res. Commun. 463, 95-101 (2015).

147. Goel, S. et al. Normalization of the vasculature for treatment of cancer and other diseases. Physiol. Rev. 91, 1071-1121 (2011).

148. Huang, Y., Goel, S., Duda, D., Fukumura, D. \& Jain, R. Vascular normalization as an emerging strategy to enhance cancer immunotherapy. Cancer Res. $\mathbf{7 3}$ 2943-2948 (2013).

149. Manegold, C. et al. The potential of combined immunotherapy and antiangiogenesis for the synergistic treatment of advanced NSCLC. J. Thorac Oncol. 12, 194-207 (2017)

150. Ott, P. A., Hodi, F. S., Kaufman, H. L., Wigginton, J. M. \& Wolchok, J. D. Combination immunotherapy: a road map. J. Immunother. Cancer 5, e16 (2017)

151. Maude, S. L. et al. Chimeric antigen receptor T cells for sustained remissions in leukemia. N. Engl. J. Med. 371, 1507-1517 (2014).

152. Singh, R. \& Paterson, Y. Immunoediting sculpts tumor epitopes during immunotherapy. Cancer Res. 67, 1887-1892 (2007).

153. Zhang, Q. et al. Chimeric antigen receptor-modified T Cells inhibit the growth and metastases of established tissue factor-positive tumors in NOG mice. Oncotarget 8, 9488-9499 (2017).

154. Feng, $K$ et al. Chimeric antigen receptor-modified $T$ cells for the immunotherapy of patients with EGFR-expressing advanced relapsed/refractory non-small cell lung cancer. Sci. China Life Sci. 59, 468-479 (2016).

155. Lee, L. et al. NY-ESO-1 may be a potential target for lung cancer immunotherapy. Cancer J. Sci. Am. 5, 20-25 (1999). 